\title{
On a new multivariate IFR ageing notion based on the standard construction
}

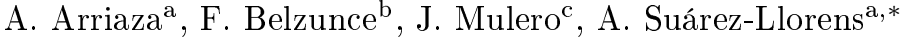 \\ ${ }^{a}$ Dpto. Estadística e Investigación Operativa, Universidad de Cádiz, Facultad de Ciencias, Campus Universitario \\ Río San Pedro s/n, 11510 Puerto Real, Cádiz, SPAIN \\ ${ }^{b}$ Dpto. Estadística e Investigación Operativa, Universidad de Murcia, Facultad de Matemáticas, Campus de \\ Espinardo, 30100 Espinardo (Murcia), SPAIN \\ ${ }^{c}$ Dpto. Estadística e Investigación Operativa, Universidad de Alicante, Facultad de Ciencias, Apartado de correos \\ 99, 03080, Alicante, SPAIN
}

\begin{abstract}
Many criteria of ageing for random variables or vectors have been proposed in the literature over many years. For instance, a random variable is increasing in failure rate (IFR) if, and only if, it can be ordered with an exponentially distributed random variable in the univariate convex transform order proposed by van Zwet [35]. Recently, Belzunce et al. [9] introduced a multivariate generalization of the convex transform order. In this work, we proposed a new multivariate IFR notion for multivariate distributions based on comparisons in this new order with a properly defined exponentially distributed random vector. Properties, applications and illustrations of this new notion are given as well.
\end{abstract}

Keywords: Standard Construction, Multivariate Distributional Transform, Multivariate Convex Transform Order, Multivariate IFR distributions

\section{Introduction}

Notions of ageing play an important role in reliability theory, survival analysis and other fields. By ageing we mean "the phenomenon whereby an older system has a shorter remaining lifetime, in some statistical sense than a younger one" (see Bryson and Siddiqui [14]). In this context, ageing notions are usually defined through different properties of well-known functions like the survival, hazard rate and mean residual life. For example, the increasing [decreasing] failure rate (IFR [DFR]) notion is one of the most studied and can be defined in terms of the hazard rate function as we recall next.

Given a random variable $X$ with absolutely continuous distribution function $F$, survival function $\bar{F}=1-F$ and density function $f$, the hazard or failure rate function is given by $r(x)=f(x) / \bar{F}(x)$, for all $x$ such that $F(x)<1$. This is one of the basic functions in the context of reliability and survival analysis where a random variable $X$ represents the random lifetime of a unit or a mechanism and it can be considered as the rate at which a unit fails when survive up to a fixed time $x$. For

\footnotetext{
${ }^{*}$ Corresponding author

Email addresses: antoniojesus.arriaza@uca.es (A. Arriaza), belzunce@um.es (F. Belzunce), julio.mulero@ua.es (J. Mulero), alfonso.suarez@uca.es (A. Suárez-Llorens)
} 
instance, if $r(x)$ is a nondecreasing [nonincreasing] function in $x$, then $X$ is said to be IFR [DFR] (see, e.g., Barlow and Proschan [8] and Lai and Xie [22]).

As pointed out by Belzunce and Shaked [11] and Belzunce and Shaked [12], the great majority of ageing notions can be characterized by means of stochastic comparisons. In particular, some ageing notions can be stated through stochastic comparisons among the random variable and an exponential distribution. Recall that a system or unit with an exponentially distributed lifetime posses the nonageing property. More precisely, the IFR [DFR] notion can be characterized in terms of the convex transform order introduced by van Zwet [35]. Let us recall its definition.

Let $X$ and $Y$ be two random variables with interval supports (finite or infinite) and distribution functions $F$ and $G$, respectively. From now on, let us denote by $G^{-1}(u)=\inf \{x: G(x) \geq u\}$ the generalized quantile function corresponding to $Y$. It is well known that the transformation $\phi(x)=G^{-1} F(x)$ maps $X$ onto $Y$, that is,

$$
Y={ }_{s t} \phi(X) .
$$

Note that it also holds $\phi(x)=\bar{G}^{-1} \bar{F}(x)$. According to this notation, $X$ is said to be smaller than $Y$ in the convex transform order, denoted by $X \leq_{c} Y$, if $\phi(x)$ is convex in the support of $X$.

From this point of view, we can say that $Y$ is obtained through a convex transformation of the sample space of $X$. In fact, the convex transform order is closely connected with the notion of skewness. Particularly, if $X$ and $Y$ are two nonnegative random variables such that $X \leq_{c} Y$, it has been accepted (see, for instance, Arnold and Groeneveld [5]) that any measure $\gamma$ of skewness should satisfy that $\gamma(X) \leq \gamma(Y)$ or, roughly speaking, $Y$ is more right-tailed than $X$. This is the case of some well known measure like the Fisher's and Bowley's coefficients and some other measures (see MacGillivray [23]).

Under the previous assumptions, a random variable $X$ is IFR [DFR] if, and only if, it is smaller [larger] in the convex transform order than an exponentially distributed random variable $E$ with scale parameter $\lambda=1$, i.e., $E \sim \operatorname{Exp}(1)$, that is,

$$
\begin{aligned}
X \text { is IFR [DFR] } & \Leftrightarrow X \leq_{c}\left[\geq_{c}\right] E, \\
& \Leftrightarrow X \leq_{c}\left[\geq_{c}\right] a E+b, \text { for all } a>0, b \in \mathbb{R},
\end{aligned}
$$

where the second characterization follows from the well-known fact that the convex transform order is preserved by translations and positive scale changes.

Other characterizations of the IFR [DFR] notion are given in terms of stochastic comparisons of residual lives for some other stochastic orders, like the usual stochastic, hazard rate, dispersive, increasing concave and Laplace transform orders (see Belzunce and Shaked [11]).

In the multivariate case, different generalizations of the IFR [DFR] have been proposed. For instance, some of them are given in terms of multivariate extensions of the hazard rate function from a mathematical point of view (see Block and Savits [13], Johnson and Kotz [19], Rüschendorf [30] and Savits [31]), while some others are given from a dynamic version of the hazard rate function (for a recent review, the reader can look at Shaked and Shanthikumar [33]).

Even more, multivariate generalizations by means of stochastic orders can be also found. In particular, there are two different proposals. Next, we describe them (see Arias-Nicolás et al. [2], Roy [28] and Roy [29]).

On one hand, the univariate IFR [DFR] notion can be characterized by dispersive comparisons of residual lives $X_{t}=(X-t \mid X>t)$. In particular, a random variable $X$ is IFR [DFR] if, and only if, $X_{t^{\prime}} \leq_{\text {disp }} X_{t}$ whenever $0 \leq t \leq t^{\prime}$ (see Belzunce and Shaked [11] and Belzunce and Shaked 
[12] for further details). Arias-Nicolás et al. [2] introduced a multivariate IFR notion based on the previous characterization comparing multivariate residual lives in the multivariate dispersive order proposed by Fernández-Ponce and Suárez-Llorens [17]. It is worth to mention that the definition of this multivariate dispersive order involves a particular transformation which will be recalled in Section 2, by means of the standard construction.

On the other hand, Roy [28] gave the definition of a new multivariate extension of the IFR notion. Then, Roy [29] proposed a multivariate convex order in terms of convex comparisons among a certain type of conditional random variables constructed with the margins of the random vector. In the same paper, Roy [29] characterized the multivariate IFR notion proposed by Roy [28] by using jointly the multivariate convex order and a Gumbel multivariate exponentially distributed random vector. This approach is a natural extension of the characterization given in (2).

The aim of this paper is to propose a new multivariate IFR [DFR] ageing notion as a natural generalization of (2). A first approach in this sense can be seen in Mulero [24]. It is apparent that a multivariate convex order and a multivariate exponential distribution are required. On one hand, we considered the so-called mct order recently defined by Belzunce et al. [9] whose definition is based on the mapping also used by Arias-Nicolás et al. [2]. On the other hand, in contrast to the previous proposal by Roy [29] who considered an existing multivariate exponential distribution, a suitable exponentially distributed random vector is introduced. Properties and relationships of the new multivariate notion are studied and some examples related with well-known models such as normal distributions or generalized ordered statistics are provided.

The paper is organized as follows. In Section 2, we recall the definition of the multivariate convex transform order proposed by Belzunce et al. [9] and some of its properties. In Section 3, we introduce a new family of exponential random vectors and use it together with the previous multivariate convex order to define a new multivariate IFR notion and study its interpretation and properties. In Section 4, some examples related to two well known probabilistic models are presented. In Section 5, we study this notion in some distributions through different plots. Finally, in Section 6, we give a brief summary of the work.

Throughout this paper, "increasing" means "nondecreasing" and "decreasing" means "nonincreasing". Given a vector (or matrix) $\mathbf{v}$, we denote as $\mathbf{v}^{t}$ the transposition of $\mathbf{v}$. Moreover, we will denote by $=_{s t}$, the equality in law, and by $\leq_{a . s .}$, the almost surely inequality. For any random vector $\mathbf{X}$, or random variable, we will denote by $(\mathbf{X} \mid A)$ a random vector, or random variable, whose distribution is the conditional distribution of $\mathbf{X}$ given $A$.

\section{Multivariate convex orders}

Given two continuous random vectors $\mathbf{X}=\left(X_{1}, \ldots, X_{n}\right)$ and $\mathbf{Y}=\left(Y_{1}, \ldots, Y_{n}\right)$, it is possible to find a function $\boldsymbol{\Phi}$ which maps the random vector $\mathbf{X}$ onto $\mathbf{Y}$ as a generalization of (1). In particular, $\boldsymbol{\Phi}$ is constructed in terms of the standard construction of $\mathbf{Y}$ and the multivariate distributional transform of $\mathbf{X}$ which play the role of the quantile and the distribution function of the random variables, respectively. Let us recall the definitions.

The problem of defining a suitable multivariate extension of the univariate quantile function has a long history in statistics and probability. In this paper, we consider the so-called standard construction, also known as multivariate quantile transformation, which is widely used in simulation theory (we refer the reader to Arjas and Lehtonen [3], O'Brien [25], Rosenblatt [27] or Rüschendorf [30] for further information). Next, we recall the definition. 
Given a continuous random vector $\mathbf{Y}=\left(Y_{1}, \ldots, Y_{n}\right)$, its standard construction is defined as

$$
\mathbf{Q}_{\mathbf{Y}}\left(u_{1}, \ldots, u_{n}\right)=\left(Q_{\mathbf{Y}, 1}\left(u_{1}\right), Q_{\mathbf{Y}, 2}\left(u_{1}, u_{2}\right), \ldots, Q_{\mathbf{Y}, n}\left(u_{1}, \ldots, u_{n}\right)\right)
$$

where

$$
\begin{aligned}
Q_{\mathbf{Y}, 1}\left(u_{1}\right) & =G_{Y_{1}}^{-1}\left(u_{1}\right), \\
Q_{\mathbf{Y}, 2}\left(u_{1}, u_{2}\right) & =G_{\left(Y_{2} \mid Y_{1}=Q_{\mathbf{Y}, 1}\left(u_{1}\right)\right)}^{-1}\left(u_{2}\right), \\
& \vdots \\
Q_{\mathbf{Y}, n}\left(u_{1}, u_{2}, \ldots, u_{n}\right) & =G_{\left(Y_{n} \mid \cap_{j=1}^{n-1}\left\{Y_{j}=Q_{\mathbf{Y}, j}\left(u_{1}, u_{2}, \ldots, p_{j}\right)\right\}\right)}^{-1}\left(u_{n}\right),
\end{aligned}
$$

for all $u_{i} \in(0,1), i=1,2, \ldots, n$, where $G_{Z}^{-1}$ denotes the quantile function of $Z$.

It is well-known that if $\mathbf{U}=\left(U_{1}, \ldots, U_{n}\right)$ is a random vector with independent and uniformly distributed components on the interval $(0,1)$, then

$$
\mathbf{Y}={ }_{s t} \mathbf{Q}_{\mathbf{Y}}(\mathbf{U}) \text {. }
$$

On the other hand, given another continuous random vector $\mathbf{X}=\left(X_{1}, \ldots, X_{n}\right)$, its multivariate distributional transform is defined as

$$
\mathbf{D}_{\mathbf{X}}\left(x_{1}, \ldots, x_{n}\right)=\left(D_{\mathbf{X}, 1}\left(x_{1}\right), D_{\mathbf{X}, 2}\left(x_{1}, x_{2}\right), \ldots, D_{\mathbf{X}, n}\left(x_{1}, \ldots, x_{n}\right)\right),
$$

where

$$
\begin{aligned}
D_{\mathbf{X}, 1}\left(x_{1}\right) & =F_{X_{1}}\left(x_{1}\right), \\
D_{\mathbf{X}, 2}\left(x_{1}, x_{2}\right) & =F_{\left(X_{2} \mid X_{1}=x_{1}\right)}\left(x_{2}\right), \\
& \vdots \\
D_{\mathbf{X}, n}\left(x_{1}, \ldots, x_{n}\right) & =F_{\left(X_{n} \mid \cap_{j=1}^{n-1}\left\{X_{j}=x_{j}\right\}\right)}\left(x_{n}\right),
\end{aligned}
$$

for all $x_{i}$ in the support of $X_{i}$, where $F_{Z}$ denotes the distribution function of $Z$.

Again, if $\mathbf{U}=\left(U_{1}, \ldots, U_{n}\right)$ is a random vector with independent and uniformly distributed components on the interval $(0,1)$, then

$$
\mathbf{U}={ }_{s t} \mathbf{D}_{\mathbf{X}}(\mathbf{X})
$$

Given two continuous random vectors $\mathbf{X}=\left(X_{1}, \ldots, X_{n}\right)$ and $\mathbf{Y}=\left(Y_{1}, \ldots, Y_{n}\right)$, let $\mathbf{\Phi}=\mathbf{Q}_{\mathbf{Y}}$ 。 $\mathbf{D}_{\mathbf{X}}$, defined for all $\left(x_{1}, \ldots, x_{n}\right)$ in the support of $\mathbf{X}$, as

$$
\boldsymbol{\Phi}\left(x_{1}, \ldots, x_{n}\right)=\left(\boldsymbol{\Phi}_{1}\left(x_{1}\right), \boldsymbol{\Phi}_{2}\left(x_{1}, x_{2}\right), \ldots, \boldsymbol{\Phi}_{n}\left(x_{1}, x_{2}, \ldots, x_{n}\right)\right)
$$

where

$$
\begin{gathered}
\boldsymbol{\Phi}_{1}\left(x_{1}\right)=F_{Y_{1}}^{-1}\left(F_{X_{1}}\left(x_{1}\right)\right), \\
\boldsymbol{\Phi}_{2}\left(x_{1}, x_{2}\right)=F_{\left(Y_{2} \mid Y_{1}=\boldsymbol{\Phi}_{1}\left(x_{1}\right)\right)}^{-1}\left(F_{\left(X_{2} \mid X_{1}=x_{1}\right)}\left(x_{2}\right)\right), \\
\vdots \\
\boldsymbol{\Phi}_{n}\left(x_{1}, \ldots, x_{n}\right)=F_{\left(Y_{n} \mid \cap_{j=1}^{n-1}\left\{Y_{j}=\boldsymbol{\Phi}_{j}\left(x_{1}, \ldots, x_{j}\right)\right\}\right)}^{-1}\left(F_{\left(X_{n} \mid \cap_{j=1}^{n-1}\left\{X_{j}=x_{j}\right\}\right)}\left(x_{n}\right)\right),
\end{gathered}
$$

Since (3) and (5), it can be seen that the function $\mathbf{\Phi}$ maps the random vector $\mathbf{X}$ onto $\mathbf{Y}$, i.e., $\mathbf{Y}={ }_{s t} \mathbf{\Phi}(\mathbf{X})$ which is the key property of this function.

From the increasingness of both the distribution function and its inverse, $\boldsymbol{\Phi}_{i}\left(x_{1}, \ldots, x_{i}\right)$ is increasing in $x_{i}$, for all $i=1, \ldots, n$. Hence the Jacobian matrix of $\boldsymbol{\Phi}$ is always a lower triangular matrix with strictly positive diagonal elements. 
Remark 1. As a clear extension of the univariate case, Fernández-Ponce and Suárez-Llorens [17] proved in Theorem 3.1 that if we have $\mathbf{k}: \mathbb{R}^{n} \rightarrow \mathbb{R}^{n}$ such that $\mathbf{Y}={ }_{\text {st }} \mathbf{k}(\mathbf{X})$ and $\mathbf{k}$ has a lower triangular Jacobian matrix with strictly positive diagonal elements, then $\mathbf{k}$ has necessarily the form of the function $\mathbf{\Phi}$ given in (6).

As a consequence of Remark 1, if we interchange the role that play $\mathbf{X}$ and $\mathbf{Y}$ in (6), we easily obtain that the function that maps $\mathbf{Y}$ onto $\mathbf{X}$ is the inverse of the corresponding that maps $\mathbf{X}$ onto $\mathbf{Y}$, i.e.,

$$
\mathbf{Q}_{\mathbf{X}} \circ \mathbf{D}_{\mathbf{Y}}=\left(\mathbf{Q}_{\mathbf{Y}} \circ \mathbf{D}_{\mathbf{X}}\right)^{-1} .
$$

On the other hand, Belzunce et al. [9] proposed the so-called mct-order which is a generalization of the univariate convex transform order. In particular, they required the convexity of the components of $\boldsymbol{\Phi}$. Next, we recall this definition.

Definition 1. Let $\mathbf{X}=\left(X_{1}, \ldots, X_{n}\right)$ and $\mathbf{Y}=\left(Y_{1}, \ldots, Y_{n}\right)$ be two random vectors. Let $\mathbf{\Phi}=$ $\left(\boldsymbol{\Phi}_{1}, \ldots, \boldsymbol{\Phi}_{n}\right)$ be the transformation described in (6). Then, $\mathbf{X}$ is said to be smaller than $\mathbf{Y}$ in the multivariate convex transform order, denoted by $\mathbf{X} \leq_{m c t} \mathbf{Y}$, if, and only if, $\boldsymbol{\Phi}_{i}: \mathbb{R}^{i} \rightarrow \mathbb{R}$ is convex in its support, for all $i=1, \ldots, n$.

Remark 2. For two univariate random variables, $\boldsymbol{\Phi}$ is always increasing. Therefore, if $\boldsymbol{\Phi}$ is convex, its inverse is concave. Unlike the univariate case, in the multivariate case, the transformation $\boldsymbol{\Phi}$ is not necessarily increasing for all components. Hence if $\boldsymbol{\Phi}$ is a multivariate convex function, as in Definition 1, it does not necessarily imply that its inverse is concave. A straightforward computation shows that this last property holds when $\boldsymbol{\Phi}_{i}$ is increasing, for all $i=1, \ldots, n$.

\section{A new definition of multivariate IFR [DFR] distributions based on the standard construction}

In this section, a new multivariate IFR ageing notion is proposed, as a direct extension of the univariate characterization given in (2). For our purpose, a properly defined exponentially distributed random vector is introduced. Note that Corollary 2 in Belzunce et al. [9] inherently deals with this multivariate exponential distribution.

Definition 2. Let $\mathbf{Y}=\left(Y_{1}, \ldots, Y_{n}\right)$ be a random vector. Then, $\mathbf{Y}$ is said to follow a multivariate conditional exponential distribution with parameter matrix $\mathbf{A}=\left(a_{i j}\right) \in \mathbb{M}_{n \times n}$ and constant vector $\mathbf{b} \in \mathbb{R}^{n}$, denoted by $\mathbf{Y} \sim \operatorname{MCE}(\mathbf{A}, \mathbf{b})$, if $\mathbf{Y}^{t}={ }_{\text {st }} \mathbf{A} \mathbf{E}^{t}+\mathbf{b}^{t}$, where $\mathbf{A}$ is a lower triangular matrix with strictly positive diagonal elements and $\mathbf{E}=\left(E_{1}, \ldots, E_{n}\right)$ denotes a multivariate random vector with independent and identically distributed (for short, i.i.d.) exponential marginal distributions with common parameter $\lambda=1$.

Under this notation, $\mathbf{E} \sim M C E\left(\mathbf{I}_{n \times n}, \mathbf{0}_{n}\right)$, where $\mathbf{I}_{n \times n}$ and $\mathbf{0}_{n}$ represent the identity matrix and the null vector, respectively.

Remark 3. It is worth to mention that this kind of distributions have been used in reliability in other contexts. For instance, Freund [18] provided a failure mechanism of a two-component system assuming that components have constant failure rates when both are in operation. In this approach, a failure of a component does not result in its replacement, but it changes the failure rate of the non-failed component as it has a higher workload. 
Let us suppose that a system has two independent components with lifetimes $X_{i} \sim \operatorname{Exp}\left(1 / \theta_{0}\right)$, for $i=1,2$. After the failure of a component, the conditional distribution for the lifetime of the remaining component is also assumed to be exponential with scale parameter, or failure rate, $1 / \theta_{1}$. Under these assumptions, the bivariate random vector whose marginal distributions represent the first and second failures is given by $\left(T_{1}, T_{2}\right)$ such that

$$
\begin{aligned}
& T_{1}=\min \left\{X_{1}, X_{2}\right\}={ }_{s t} \operatorname{Exp}\left(2 / \theta_{0}\right), \\
& T_{2}=T_{1}+Y_{2},
\end{aligned}
$$

where $Y_{2} \sim \operatorname{Exp}\left(1 / \theta_{1}\right)$ and $T_{1}$ and $Y_{2}$ are independent. In this case, the joint density function of $\left(T_{1}, T_{2}\right)$ is given by

$$
f_{\left(T_{1}, T_{2}\right)}\left(t_{1}, t_{2}\right)=f_{T_{1}}\left(t_{1}\right) f_{\left(T_{2} \mid T_{1}=t_{1}\right)}\left(t_{2}\right)=2 \theta_{0}^{-1} \theta_{1}^{-1} \exp \left\{-2 \theta_{0}^{-1} t_{1}-\theta_{1}^{-1}\left(t_{2}-t_{1}\right)\right\},
$$

where $0 \leq t_{1} \leq t_{2}$. Just by construction, it is easy to see that $\left(T_{1}, T_{2}\right) \sim \operatorname{MCE}\left(\mathbf{A}_{2 \times 2}, \mathbf{0}_{n}\right)$ where the lower triangular matrix $\mathbf{A}=\left(a_{i j}\right) \in \mathbb{M}_{2 \times 2}$ satisfies that $a_{11}=a_{21}=\theta_{0} / 2$ and $a_{22}=\theta_{1}$.

Given $\mathbf{Y} \sim \operatorname{MCE}(\mathbf{A}, \mathbf{b})$, it is remarkable that $Y_{i}$ is always a linear combination of independent shifted exponential distributions, for all $i=1 \ldots, n$. Furthermore, the name "conditional exponential" comes from the property that the following sequential conditional distributions are shifted exponential distributions, that is,

$$
\begin{aligned}
Y_{1} & \sim a_{11} E_{1}+b_{1}, \\
\left(Y_{2} \mid Y_{1}=y_{1}\right) & \sim a_{21} \alpha_{1}+a_{22} E_{2}+b_{2}, \\
\left(Y_{3} \mid Y_{1}=y_{1}, Y_{2}=y_{2}\right) & \sim a_{31} \alpha_{1}+a_{32} \alpha_{2}+a_{33} E_{3}+b_{3}, \\
& \vdots \\
\left(Y_{n} \mid \cap_{j=1}^{n-1} Y_{j}=y_{j}\right) & \sim \sum_{j=1}^{n-1} a_{n j} \alpha_{j}+a_{n n} E_{n}+b_{n},
\end{aligned}
$$

where the conditional distributions are well defined. It is not difficult to see that

$$
\left(\alpha_{1}, \ldots, \alpha_{n-1}\right)^{t}=\mathbf{A}_{n-1}^{-1}\left(y_{1}-b_{1}, \ldots, y_{n-1}-b_{n-1}\right)^{t},
$$

where $\mathbf{A}_{n-1}$ is a submatrix formed by deleting the $n$-th row and $n$-th column of $\mathbf{A}$. Since (8), the joint density function of a multivariate conditional exponential distribution can be easily computed.

Remark 4. Let $\mathbf{Y}_{1} \sim \operatorname{MCE}\left(\mathbf{A}_{1}, \mathbf{b}_{1}\right)$ and $\mathbf{Y}_{2} \sim \operatorname{MCE}\left(\mathbf{A}_{2}, \mathbf{b}_{2}\right)$ be two multivariate conditional exponential distributions. With a straightforward computation, it can be showed that

$$
\mathbf{Y}_{2}^{t}={ }_{s t} \mathbf{A}_{2} \mathbf{A}_{1}^{-1}\left(\mathbf{Y}_{1}-\mathbf{b}_{1}\right)^{t}+\mathbf{b}_{2}^{t} .
$$

Just taking into account that $\mathbf{A}_{1}^{-1}$ is a lower triangular matrix with strictly positive diagonal elements, we have that $\mathbf{A}_{2} \mathbf{A}_{1}^{-1}$ is also a lower triangular matrix with strictly positive diagonal elements. Therefore, from Proposition 2 in Belzunce et al. [9], all multivariate conditional exponential distributions are equal in the mct-order, i.e., $\mathbf{Y}_{1}={ }_{m c t} \mathbf{Y}_{2}$.

From the definition of the multivariate conditional exponential distribution and clearly inspired in (2), we propose a new multivariate IFR ageing notion based on the mct-order. 
Definition 3. Let $\mathbf{X}=\left(X_{1}, \ldots, X_{n}\right)$ be an absolutely continuous random vector. Then, $\mathbf{X}$ is said to be mct-IFR $[$ mct-DFR $]$ if there exists a particular multivariate conditional exponential distribution, $\mathbf{Y} \sim \operatorname{MCE}(\mathbf{A}, \mathbf{b})$, such that $\mathbf{X} \leq_{m c t}\left[\geq_{m c t}\right] \mathbf{Y}$. We will call the random vector $\mathbf{Y}$ an upper bound exponential distribution associated with $\mathbf{X}$.

Remark 5. Just by taking $n=1$, Definition 3 is an extension of the univariate characterization given in (2). From Remark 2, it is worth to mention that checking the convexity of $\mathbf{\Phi}$ is not equivalent to check the concavity of $\boldsymbol{\Phi}^{-1}$, for $n>1$. Therefore, if we interchange the role that play $\mathbf{X}$ and $\mathbf{Y}$ in Definition 3 and we require concavity instead of convexity it will lead us to a complementary ageing definition as we will see later in Counterexample 2.

Remark 6. Before studying the main properties, note that if $\mathbf{X}$ is mct-IFR, then there exists $\mathbf{Y} \sim \operatorname{MCE}(\mathbf{A}, \mathbf{b})$ such that $\mathbf{X} \leq_{m c t} \mathbf{Y}$. Therefore, from Remark 4 , we easily obtain that $\mathbf{X} \leq_{m c t}$ $\mathbf{Y}={ }_{\text {mct }} \mathbf{Y}^{\prime}$ for any $\mathbf{Y}^{\prime} \sim \operatorname{MCE}\left(\mathbf{A}^{\prime}, \mathbf{b}^{\prime}\right)$. At this point, it is natural to wonder if the previous result implies that $\mathbf{X} \leq_{m c t} \mathbf{Y}^{\prime}$. The answer is not, due to fact that the multivariate transform convex order is not transitive in general, see Example 3 in Belzunce et al. [9]. Hence if $\mathbf{X}$ is mct-IFR, we can not conclude that it is ordered in the mct-order sense with any multivariate conditional exponential distribution unlike the univariate case.

This is not surprising if we recall that the function $\mathbf{\Phi}$ that maps a random vector onto another one depends on the standard construction and this one is related to the dependence structure of the corresponding random vectors, for more details about that dependency see Fernández-Ponce et al. [15]. However, Proposition 4 in Belzunce et al. [9] states conditions to ensure the transitivity. In particular, $\mathbf{X} \leq_{m c t} \mathbf{Y}^{\prime}$ holds if the affine function given in Remark $4, A^{\prime} A^{-1}(\mathbf{y}-\mathbf{b})^{t}+\left(\mathbf{b}^{\prime}\right)^{t}$, which maps $\mathbf{Y}$ onto $\mathbf{Y}^{\prime}$ is increasing, i.e., the matrix $A^{\prime} A^{-1}$ has nonnegative coefficients.

Finally, by changing the constant vector $\mathbf{b}$, the upper bound distribution is not unique.

Next, some desirable properties associated with Definition 3 are presented. First, we show that random vectors with independent IFR [DFR] marginal distributions are mct-IFR [mct-DFR]. Therefore, a random vector with independent components inherits the univariate ageing properties of the marginal distributions. It is worth to mention that most of the multivariate extensions of the IFR notion satisfy this kind of property (see, among others, Block and Savits [13], Johnson and Kotz [19], Savits [31], Arias-Nicolás et al. [2], Roy [28], Roy [29] and Fernández-Ponce et al. [16]).

Proposition 1. Let $\mathbf{X}=\left(X_{1}, \ldots, X_{n}\right)$ be an absolutely continuous random vector with independent components. Then, $\mathbf{X}$ is mct-IFR $[$ mct-DFR $]$ if, and only if, $X_{i}$ is IFR $[D F R]$, for all $i=1, \ldots, n$.

Proof. We just need to prove that $\mathbf{E} \sim \operatorname{MCE}\left(\mathbf{I}_{n \times n}, \mathbf{0}_{n}\right)$ is an upper bound exponential distribution associated with $\mathbf{X}$. Due to the fact that $\mathbf{X}$ and $\mathbf{E}$ share the same independence copula, just using Theorem 3 in Belzunce et al. [9], we have that $\mathbf{X} \leq_{m c t}\left[\geq_{m c t}\right] \mathbf{E}$ holds if, and only if, $X_{i} \leq_{c}\left[\geq_{c}\right] E_{i}$, for all $i=1 \ldots, n$. Since (2), the last condition holds if, and only if, $X_{i}$ 's are IFR [DFR], for all $i=1 \ldots, n$.

Some results that can help us to provide many possible examples are stated in Propositions 2 and 3. Their proofs are an immediate consequence of the transitivity property of the mct-order described in Proposition 4 in Belzunce et al. [9] and have been omitted.

Proposition 2. Let $\mathbf{X}=\left(X_{1}, \ldots, X_{n}\right)$ be an absolutely continuous mct-IFR distribution and let $\mathbf{Y} \sim \operatorname{MCE}(\mathbf{A}, \mathbf{b})$ be an upper bound exponential distribution associated with $\mathbf{X}$. Let $\mathbf{\Phi}=\mathbf{Q}_{\mathbf{Y}} \circ \mathbf{D}_{\mathbf{X}}$ the transformation that maps $\mathbf{X}$ onto $\mathbf{Y}$. If $\mathbf{\Phi}(\mathbf{x})$ is increasing for all $\mathbf{x}$ in the support of $\mathbf{X}$, then any random vector $\mathbf{X}^{\prime}$ such that $\mathbf{X}^{\prime} \leq_{m c t} \mathbf{X}$ is also met-IFR. 
Proposition 3. Let $\mathbf{X}=\left(X_{1}, \ldots, X_{n}\right)$ be an absolutely continuous mct-DFR distribution, then any random vector $\mathbf{X}^{\prime}$ such that $\mathbf{X} \leq_{m c t} \mathbf{X}^{\prime}$ is also mct-DFR whenever the transformation $\mathbf{\Phi}=\mathbf{Q}_{\mathbf{X}^{\prime}} \circ \mathbf{D}_{\mathbf{X}}$ that maps $\mathbf{X}$ onto $\mathbf{X}^{\prime}$ is increasing for all $\mathbf{X}$ in the support of $\mathbf{X}$.

Note that given a random vector $\mathbf{X}$, it is remarkable that fixing a common copula, a constructive way of finding $\mathbf{X}^{\prime}$ such that $\mathbf{X} \leq_{m c t} \mathbf{X}^{\prime}$ or $\mathbf{X}^{\prime} \leq_{m c t} \mathbf{X}$ is described in Section 3 in Belzunce et al. [9]. It is clear that this fact allows us to construct many examples using the previous propositions.

Now, we present some examples related to linear transformations where we can also apply the previous propositions.

Example 1. Let $\mathbf{X}=\left(X_{1}, \ldots, X_{n}\right)$ be an absolutely continuous mct-IFR distribution satisfying the conditions in Proposition 2. Let $\mathbf{X}^{\prime}=\mathbf{X B}^{t}$ be a linear transformation of $\mathbf{X}$, where $\mathbf{B}$ is a lower triangular matrix with strictly positive diagonal elements. From Proposition 2 in Belzunce et al. [9] $\mathbf{X}^{\prime}={ }_{m c t} \mathbf{X}$, in particular $\mathbf{X}^{\prime} \leq_{m c t} \mathbf{X}$ holds. Therefore, using Proposition $2 \mathbf{X}^{\prime}$ is mct-IFR.

Example 2. Let $\mathbf{X}=\left(X_{1}, \ldots, X_{n}\right)$ be an absolutely continuous mct-DFR distribution. Let $\mathbf{X}^{\prime}=$ $\mathbf{X B}^{t}$ be a linear transformation of $\mathbf{X}$, where $\mathbf{B}=\{a i j\}$ is a lower triangular matrix such that aij $\geq 0$ with strictly positive diagonal elements. From Proposition 2 in Belzunce et al. [9] $\mathbf{X}={ }_{m c t} \mathbf{X}^{\prime}$, in particular $\mathbf{X} \leq_{m c t} \mathbf{X}^{\prime}$ holds and, using Remark 1 the transformation $\mathbf{\Phi}$ that maps $\mathbf{X}$ onto $\mathbf{X}^{\prime}$ has the form $\mathbf{\Phi}(\mathbf{X})=\mathbf{X B}^{t}$, which is trivially increasing. Therefore, using Proposition $3 \mathbf{X}^{\prime}$ is mct-DFR.

The previous examples will allow us to interpret an interesting model as a generalization of the model by Freund [18].

Example 3. In the literature, a multivariate Weibull distribution in the sense of Freund can be found. This is a more general situation than the exponential one shown in Remark 3 (for further information, see Rinne [26] and references therein). For the sake of simplicity, we again restrict to a system with two components as in Remark 3.

Let $X_{i} \sim W\left(1 / \theta_{0}, k_{0}\right), i=1,2$, be two independent components which follow, when both are in operation, the same Weibull distribution with shape and scale parameters $k_{0}>0$ and $\theta_{0}>0$, respectively, where we recall that

$$
F_{W\left(1 / \theta_{0}, k_{0}\right)}(t)=1-\exp \left\{-\left(\theta_{o}^{-1} t\right)^{k_{0}}\right\}, \quad \forall x \geq 0 .
$$

After the failure of a component, the remaning lifetime of the other one is still Weibull. In particular, the shape parameter $k_{1}$ maybe remains as $k_{0}$, but the scale parameter $\theta_{1}$ is not generally equal to $\theta_{0}$. Analogously to Example 3, the bivariate random vector whose marginal distributions represent the first and second failures is given by $\left(T_{1}, T_{2}\right)$ such that

$$
\begin{aligned}
& T_{1}=\min \left\{X_{1}, X_{2}\right\}=s t W\left(\sqrt[k]{2} / \theta_{0}, k_{0}\right), \\
& T_{2}=T_{1}+M_{2},
\end{aligned}
$$

where $M_{2} \sim W\left(1 / \theta_{1}, k_{1}\right)$ and $T_{1}$ and $M_{2}$ are independent. Just by construction is easy to observe that $\left(T_{1}, T_{2}\right)=\left(W_{1}, W_{2}\right) \mathbf{B}^{t}$ such that $W_{1}={ }_{s t} W\left(\sqrt[k_{0}]{2} / \theta_{0}, k_{0}\right)$ and $W_{2}={ }_{s t} W\left(1 / \theta_{1}, k_{1}\right)$ are independent and the lower triangular matrix $\mathbf{B}=\left(b_{i j}\right) \in \mathbb{M}_{2 \times 2}$ satisfies that $b_{11}=b_{21}=b_{22}=1>0$.

From Proposition 1, $\left(W_{1}, W_{2}\right)$ is mct-IFR [mct-DFR] if, and only if, $W_{i}$, for $i=1,2$ is IFR $[D F R]$, that is, $k_{i}>[<] 1$, for $i=1,2$. In case $W_{i}$ is IFR, for $i=1,2$, it easily follows, from Example 1, that $\left(T_{1}, T_{2}\right)$ is mct-IFR. On the other hand, if $W_{i}$ is DFR, for $i=1,2$, taking into account that the function which maps $\left(W_{1}, W_{2}\right)$ onto $\left(T_{1}, T_{2}\right)$ is increasing it follows using Example 2 that $\left(T_{1}, T_{2}\right)$ is mct-IFR. Therefore, if $k_{0}, k_{1}>[<] 1,\left(T_{1}, T_{2}\right)$ is mct-IFR $[$ mct-DFR]. 
Remark 7. Just recalling that the definition of the mct-order depends on the standard construction which, in turn, is based on sequential conditional distributions, it is not surprising that Definition 3 is not invariant under permutation of the marginal distributions. Let us consider the following example: let $X$ and $Y$ be two independent DFR univariate random variables. From Proposition 1 , it can be seen that the random vector $\mathbf{X}=\left(X_{1}, X_{2}\right)$ is mct-DFR. Now, if we consider the lower triangular matrix $\mathbf{A}=\left(a_{i j}\right) \in \mathbb{M}_{2 \times 2}$ such that $a_{11}=a_{21}=a_{22}=1$, using Example 2, we have that $\mathbf{X A}^{t}=\left(X_{1}, X_{1}+X_{2}\right)$ is also mct-DFR. However, in general, we cannot expect the mct-DFR property for the random vector $\left(X_{1}+X_{2}, X_{1}\right)$ as can be seen using the following reasoning: if we assume that $\left(X_{1}+X_{2}, X_{1}\right)$ is mct-DFR, using Theorem 1 the sum $X_{1}+X_{2}$ is also DFR. Since we have not considered any restriction for $X_{1}$ and $X_{2}$, our first assumption is in contradiction with the well-known fact that the sum of two independent DFR random variables is not necessarily DFR. The above drawback naturally arises in the multivariate case, where several notions that are not invariant under permutation can be found. For instance, most of the dispersive orders defined in Belzunce et al. [10] and the multivariate ageing notions defined in Arias-Nicolás et al. [2] and Fernández-Ponce et al. [16] depends on the permutation of the components. The notion of history defined in Shaked and Shanthikumar (1991) also reflects the effect of the time over the failure probability of the components where these histories depend on the permutation of the components. Of course, Definition 3 can be restricted just for tandom vectors that are mct-IFR [mct-DFR] for any permutation of the marginal distributions. For example, by Proposition 1, a random vector with independent IFR [DFR] components is mct-IFR [mct-DFR] for any permutation of their marginal distributions, and it will be shown in Theorem 2 that this is also the case of the multivariate normal distribution. We can also restrict our study to the interesting case of exchangeable distributions, see Spizzichino [34], where the effect of the permutations is avoided. Far from be a drawback, there are many real situations where a particular permutation could determine a different aging property. In the context of computational engineering, situations where some components are "more relevant" can be easily encountered. For instance, the sequence of multiple components failure $\{C P U, R A M$ card, display card, monitor\} could have different ageing properties than that by \{monitor, display card, CPU, RAM card\}. Another situation of interest is the case where we consider increasingly ordered sequences of failure times. This is the case of the usual order statistics in which, given the failure times of $n$ independent components, the random vector of order statistics is the random vector of the increasingly ordered failure times. In other situations we have the sequence of failure times of unit subjected to a repair policy. For example in a unit subjected to a minimal repair policy the failure times are increasingly ordered, and correspond to the epoch times of nonhomogenepous Poisson process. Additional examples will be provided in Section 4 as particular cases of the model of generalized order statistics.

The following result shows that if a random vector $\mathbf{X}$ is mct-IFR [mct-DFR], then the sequential conditional distributions associated with $\mathbf{X}$ are univariate IFR [DFR].

Theorem 1. Let $\mathbf{X}$ be a mct-IFR $[$ mct-DFR $]$ distribution. Then,

$$
X_{1} \text { is } \operatorname{IFR}[D F R] \text {, }
$$

and the conditional distribution

$$
\left(X_{i} \bigcap_{j=1}^{i-1}\left\{X_{j}=Q_{\mathbf{X}, j}\left(u_{1}, \ldots, u_{j}\right)\right\}\right) \text { is IFR }[D F R],
$$

for all $i=2, \ldots, n$ and $u_{j}$ such that $0<u_{j}<1, j=1, \ldots, i-1$. 
Proof. Let $\mathbf{Y} \sim \operatorname{MCE}(\mathbf{A}, \mathbf{b})$ be the corresponding upper bound exponential distribution associated with $\mathbf{X}$. From Proposition 1 in Belzunce et al. [9], we have that

$$
\begin{gathered}
X_{1} \leq_{c}\left[\geq_{c}\right] Y_{1}, \\
\left(X_{i} \mid \bigcap_{j=1}^{i-1}\left\{X_{j}=Q_{\mathbf{X}, j}\left(u_{1}, \ldots, u_{j}\right)\right\}\right) \leq_{c}\left[\geq_{c}\right]\left(Y_{i} \mid \bigcap_{j=1}^{i-1}\left\{Y_{j}=Q_{\mathbf{y}, j}\left(u_{1}, \ldots, u_{j}\right)\right\}\right),
\end{gathered}
$$

for all $i=2, \ldots, n$ and $u_{j}$ such that $0<u_{j}<1, j=1, \ldots, i-1$. The proof follows directly just taking in account that all sequential conditional distributions associated with $\mathbf{Y}$ are univariate shifted exponential distributions, see (8), and using (2).

The previous result also shows that, when we consider a particular permutation of the components, the time of a component is IFR [DFR] for any previous sequence of failure times. This is very common in reliability where most of the MIFR [MDFR] definitions given in the literature depend either on the stochastic comparison or the comparison of the hazard rate given some history for the components. In general a history for a set of components is any event where we know the identity of the components that are still alive and the failure times for the components that have failed. In our case, the events on which we consider the conditional distribution have the form $\bigcap_{j=1}^{i-1}\left\{X_{j}=Q_{\mathbf{X}, j}\left(u_{1}, \ldots, u_{j}\right)\right\}$. For further research, this fact could help to relate our new ageing notion to previous notions in the literature.

Next, we show that the reciprocal of Theorem 1 is not true in general.

Counterexample 1. Let $\mathbf{X}=\left(X_{1}, X_{2}\right) \sim\left(E_{1}, E_{1} E_{2}\right)$ be a bivariate random vector such that $E_{1}$ and $E_{2}$ are two independent exponential distributions with scale parameter $\lambda=1$. A straightforward computation shows that

$$
\begin{aligned}
X_{1} & \sim \operatorname{Exp}(1), \\
\left(X_{2} \mid X_{1}=x_{1}\right) & \sim \operatorname{Exp}\left(\frac{1}{x_{1}}\right), \text { for all } x_{1} \in(0, \infty),
\end{aligned}
$$

which means that all sequential conditional distributions are constant hazard rate, i.e., they are both IFR and DFR. However, $\mathbf{X}$ is neither mct-IFR nor mct-DFR distribution. First, we will prove that $\mathbf{X}$ is not mct-IFR. Without loss of generality, we consider $\boldsymbol{Y}=\left(Y_{1}, Y_{2}\right) \sim M C E\left(\boldsymbol{A}_{2 \times 2}, \boldsymbol{O}_{2}\right)$. From expression (8) for $n=2$, we easily obtain that

$$
\begin{aligned}
G_{Y_{1}}^{-1}\left(u_{1}\right) & =-a_{11} \log \left(1-u_{1}\right), \\
G_{\left(Y_{2} \mid Y_{1}=y_{1}\right)}^{-1}\left(u_{2}\right) & =\frac{a_{21}}{a_{11}} y_{1}-a_{22} \log \left(1-u_{2}\right) .
\end{aligned}
$$

Therefore, using jointly (9) and (10) it is apparent that

$$
\begin{aligned}
\boldsymbol{\Phi}_{1}\left(x_{1}\right) & =G_{Y_{1}}^{-1}\left(F_{X_{1}}\left(x_{1}\right)\right)=a_{11} x_{1}, \\
\boldsymbol{\Phi}_{2}\left(x_{1}, x_{2}\right) & =G_{\left(Y_{2} \mid Y_{1}=\boldsymbol{\Phi}_{1}\left(x_{1}\right)\right)}^{-1}\left(F_{\left(X_{2} \mid X_{1}=x_{1}\right)}\left(x_{2}\right)\right)=a_{21} x_{1}+a_{22} \frac{x_{2}}{x_{1}} .
\end{aligned}
$$

It is clear that $\boldsymbol{\Phi}_{1}$ is an affine function. However, computing the Hessian matrix of $\boldsymbol{\Phi}_{2}$, we obtain that

$$
H_{\boldsymbol{\Phi}_{2}\left(x_{1}, x_{2}\right)}=\left(\begin{array}{cc}
2 a_{22} \frac{x_{2}}{x_{1}^{3}} & -\frac{a_{22}}{x_{1}^{2}} \\
-\frac{a_{22}}{x_{1}^{2}} & 0
\end{array}\right),
$$


where the principal minors are given by

$$
\begin{aligned}
& \delta_{1}=2 a_{22} \frac{x_{2}}{x_{1}^{3}}>0, \text { for all } x_{1}, x_{2} \in \mathbb{R}^{+}, \\
& \delta_{2}=-\frac{a_{22}^{2}}{x_{1}^{4}}<0, \text { for all } x_{1} \in \mathbb{R}^{+} .
\end{aligned}
$$

From Sylvester criterion and just observing that the second leading principal minor is always negative, we have that $\boldsymbol{\Phi}_{2}$ is neither a concave nor convex function. Therefore, there exists no bivariate exponential conditional distribution $\mathbf{Y} \sim \operatorname{MCE}\left(\mathbf{A}_{2 \times 2}, \mathbf{b}_{2}\right)$ such that $\mathbf{X} \leq_{m c t} \mathbf{Y}$.

In order to prove that $\mathbf{X}$ is not mct-DFR either, we now compute the function which maps $\boldsymbol{Y}$ onto $\boldsymbol{X}$. From (7), this function $\mathbf{\Phi}^{-1}$ is given by

$$
\begin{aligned}
F_{X_{1}}^{-1}\left(G_{Y_{1}}\left(y_{1}\right)\right)=\boldsymbol{\Phi}_{1}^{-1}\left(y_{1}\right) & =\frac{1}{a_{11}} y_{1}, \\
F_{\left(X_{2} \mid X_{1}=F_{X_{1}}^{-1}\left(G_{Y_{1}}\left(y_{1}\right)\right)\right)}^{-1}\left(G_{\left(Y_{2} \mid Y_{1}=y_{1}\right)}\left(y_{2}\right)\right)=\boldsymbol{\Phi}_{2}^{-1}\left(y_{1}, y_{2}\right) & =\frac{1}{a_{11} a_{22}} y_{1} y_{2}-\frac{a_{21}}{a_{11}^{2} a_{22}} y_{1}^{2} .
\end{aligned}
$$

Under a similar argument, the Hessiam matrix of (11) is given by

$$
H_{\boldsymbol{\Phi}_{2}^{-1}\left(y_{1}, y_{2}\right)}=\left(\begin{array}{cc}
-\frac{2 a_{21}}{a_{11}^{2} a_{22}} & \frac{1}{a_{11} a_{22}} \\
\frac{1}{a_{11} a_{22}} & 0
\end{array}\right),
$$

where the second leading principal minor is always negative. Therefore, there exists no bivariate exponential conditional distribution $\boldsymbol{Y} \sim \operatorname{MCE}\left(\boldsymbol{A}_{2 \times 2}, \boldsymbol{b}_{2}\right)$ such that $\boldsymbol{Y} \leq_{m c t} \boldsymbol{X}$.

We finalize the exposure of the main properties recalling Remark 5 . In particular, the next counterexample shows that replacing concavity instead of convexity in Definition 3 leads in practice to a complementary ageing notion.

Counterexample 2. Let us consider $\mathbf{X}=\left(X_{1}, X_{2}\right)$ be a classical bivariate Pareto distribution, denoted by $\mathbb{P}\left(\alpha_{1}, \alpha_{2}, a\right)$, with survival function given by

$$
\bar{F}\left(x_{1}, x_{2}\right)=\left(\frac{x_{1}}{\alpha_{1}}+\frac{x_{2}}{\alpha_{2}}-1\right)^{-a},
$$

where $x_{i} \geq \alpha_{i}>0, a>0$. It is well known that marginal distributions of $\mathbf{X}$ are distributed as univariate Pareto distributions with a common shape parameter, i.e., $X_{i} \sim \mathbb{P}\left(\alpha_{i}, a\right)$, having distribution functions $F_{X_{i}}\left(x_{i}\right)=1-\left(\frac{\alpha_{i}}{x_{i}}\right)^{a}, i=1,2$. Just computing the conditional density, it can be seen that

$$
F_{\left(X_{2} \mid X_{1}=x_{1}\right)}\left(x_{2}\right)=1-\left(\frac{\alpha_{2} x_{1}}{\alpha_{2} x_{1}+\alpha_{1} x_{2}-\alpha_{1} \alpha_{2}}\right)^{(a+1)} .
$$

Without loss of generality, let $\boldsymbol{Y}=\left(Y_{1}, Y_{2}\right) \sim M C E\left(\boldsymbol{A}_{2 \times 2}, \boldsymbol{O}_{2}\right)$ be an exponential conditional distribution. Therefore, from (10), we have that the function $\mathbf{\Phi}$ that maps $\mathbf{X}$ to $\mathbf{Y}$ is given by

$$
\begin{aligned}
\boldsymbol{\Phi}_{1}\left(x_{1}\right) & =-a_{11} a \log \left(\frac{\alpha_{1}}{x_{1}}\right) \\
\boldsymbol{\Phi}_{2}\left(x_{1}, x_{2}\right) & =-a_{21} a \log \left(\frac{\alpha_{1}}{x_{1}}\right)-a_{22}(a+1) \log \left(\frac{\alpha_{2} x_{1}}{\alpha_{2} x_{1}+\alpha_{1} x_{2}-\alpha_{1} \alpha_{2}}\right) .
\end{aligned}
$$


On the other hand, from (7), the function $\mathbf{\Phi}^{-1}$ that maps $\mathbf{Y}$ to $\mathbf{X}$ is given by

$$
\begin{aligned}
\boldsymbol{\Phi}_{1}^{-1}\left(y_{1}\right) & =\alpha_{1} \exp \left\{\frac{y_{1}}{a_{11} a}\right\} \\
\boldsymbol{\Phi}_{2}^{-1}\left(y_{1}, y_{2}\right) & =\alpha_{2}-\alpha_{2} \exp \left\{\frac{y_{1}}{a_{11} a}\right\}+\alpha_{2} \exp \left\{\frac{a_{22}(a+1)-a_{21} a}{a_{11} a_{22} a(a+1)} y_{1}+\frac{1}{a_{22}(a+1)} y_{2}\right\} .
\end{aligned}
$$

From (2) and due to the well known fact that the classical univariate Pareto distribution is DFR, $\mathbf{\Phi}_{1}^{-1}$ is an increasing convex function and, from Remark $2, \boldsymbol{\Phi}_{1}$ is a concave function. Hence $\mathbf{X}$ can not be mct-IFR, which is consistent with the univariate ageing property. Just taking derivatives, it is not difficult to see that the Hessian matrices of $\boldsymbol{\Phi}_{2}$ and $\boldsymbol{\Phi}_{2}^{-1}$ are given by

$$
H_{\mathbf{\Phi}_{2}\left(x_{1}, x_{2}\right)}=\left(\begin{array}{cc}
{\left[a_{22}(a+1)-a_{21} a\right] \frac{1}{x_{1}^{2}}-a_{22}(a+1) \alpha_{2}^{2} h} & -a_{22}(a+1) \alpha_{1} \alpha_{2} h \\
-a_{22}(a+1) \alpha_{1} \alpha_{2} h & -a_{22}(a+1) \alpha_{1}^{2} h
\end{array}\right)
$$

where $h=\left(\alpha_{1} x_{2}+\alpha_{2} x_{1}-\alpha_{1} \alpha_{2}\right)^{-2}$ and

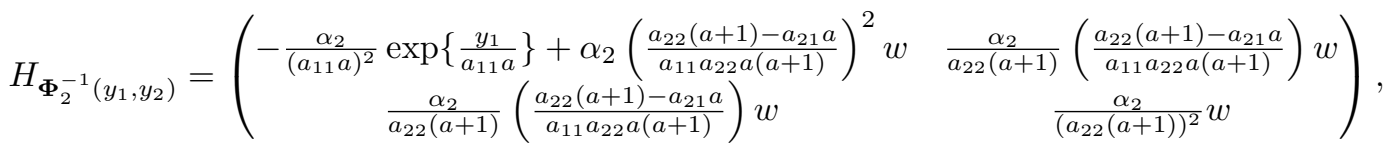

respectively, where $w=\exp \left\{\frac{a_{22}(a+1)-a_{21} a}{a_{11} a_{22} a(a+1)} y_{1}+\frac{1}{a_{22}(a+1)} y_{2}\right\}$.

It is proven that the second leading minor of $H_{\Phi_{2}^{-1}\left(y_{1}, y_{2}\right)}$ is given by

$$
\delta_{2}=-\left(\frac{\alpha_{2}}{a_{11} a_{22} a(a+1)}\right)^{2} \exp \left\{\frac{y_{1}}{a_{11} a}\right\} w<0, \text { for all } y_{1} \geq 0 .
$$

Therefore, as a direct consequence of the Sylvester criterion, $\boldsymbol{\Phi}_{2}^{-1}$ is neither concave nor convex. Hence $\mathbf{X}$ is not mct-DFR. Therefore, $\mathbf{X}$ is neither mct-IFR nor mct-DFR in the sense of Definition 3. However, just taking $a_{11}=a_{22}=1$ and $a_{21}=(a+2) / a$, the concavity of $\boldsymbol{\Phi}$ easily holds, which is a clear generalization of the univariate case.

\section{Applications and examples}

Next, two examples of known probabilistic models that satisfy the new multivariate ageing property are provided.

\subsection{Multivariate normal distributions}

The univariate normal distribution is a classical example of IFR. Next, we prove that a multivariate normal distribution is mct-IFR.

Theorem 2. Let $\mathbf{X} \sim N_{n}(\mu, \boldsymbol{\Sigma})$ be a multivariate normal distribution. Then, $\mathbf{X}$ is mct-IFR.

Proof. We just need to prove that $\mathbf{E} \sim \operatorname{MCE}\left(\mathbf{I}_{n \times n}, \mathbf{0}_{n}\right)$ is an upper bound exponential distribution associated with $\mathbf{X}$. From Example 2 in Belzunce et al. [9], we obtain that $\mathbf{X}={ }_{m c t} \mathbf{Z}$, where $\mathbf{Z} \sim N_{n}\left(\mathbf{I}_{n \times n}, \mathbf{0}\right)$. Due to the fact that $\mathbf{Z}$ and $\mathbf{E}$ share the same independence copula, just using Theorem 1 in Belzunce et al. [9], $\mathbf{Z} \leq_{m c t}\left[\geq_{m c t}\right] \mathbf{E}$ holds if, and only if, $Z_{i} \leq_{c}\left[\geq_{c}\right] E_{i}$ holds, for all $i=1 \ldots, n$. Since (2), the last condition holds from the well-known fact that univariate normal distributions are IFR. The proof concludes just using Corollary 1 in Belzunce et al. [9]. 


\subsection{Generalized order statistics}

The model of a random vector with ordered components arises in natural way when we arrange in increasing order a set of observations from a random variable. Another example is the case of epoch times of a counting process, like the case of a nonhomogeneous Poisson process. Epoch times of nonhomogeneous Poisson processes can be introduced as record values of a proper sequence of random variables, which is another typical example of ordered data. Given the similarity of several results for order statistics and record values, Kamps [20] introduced the model of generalized order statistics. This model provides a unified approach to study order statistics and record values, and several other models of ordered data. Next, we recall the definition of generalized order statistics following Kamps [20] and Kamps [21].

Definition 4. Let $n \in \mathbb{N}, k \geq 1, m_{1}, \ldots, m_{n-1} \in \mathbb{R}, M_{r}=\sum_{j=r}^{n-1} m_{j}, 1 \leq r \leq n-1$, be parameters such that $\gamma_{r}=k+n-r+M_{r} \geq 1$ for all $r \in 1, \ldots, n-1$, and let $\widetilde{m}=\left(m_{1}, \ldots, m_{n-1}\right)$, if $n \geq 2$ $(\tilde{m} \in \mathbb{R}$ arbitrary, if $n=1)$. We call uniform generalized order statistics to the random vector $\left(U_{(1, n, \tilde{m}, k)}, \ldots, U_{(n, n, \tilde{m}, k)}\right)$ with joint density function

$$
h\left(u_{1}, \ldots, u_{n}\right)=k\left(\prod_{j=1}^{n-1} \gamma_{j}\right)\left(\prod_{j=1}^{n-1}\left(1-u_{j}\right)^{m_{j}}\right)\left(1-u_{n}\right)^{k-1}
$$

on the cone $0 \leq u_{1} \leq \cdots \leq u_{n} \leq 1$. Now, given a distribution function $F$, we call generalized order statistics based on $F$ to the random vector

$$
\left(X_{(1, n, \tilde{m}, k)}, \ldots, X_{(n, n, \tilde{m}, k)}\right)=\left(F^{-1}\left(U_{(1, n, \tilde{m}, k)}\right), \ldots, F^{-1}\left(U_{(n, n, \tilde{m}, k)}\right)\right) .
$$

Theorem 3. Let $\mathbf{X}$ be the vector of generalized order statistics based on the distribution function $F$ of a random variable $X$. Then, $\mathbf{X}$ is mct-IFR $[$ mct-DFR] if, and only if, $X$ is IFR $[D F R]$.

Proof. Let $\mathbf{Y}$ be a random vector of generalized order statistics based on $E \sim \operatorname{Exp}(1)$, with the same parameters than $\mathbf{X}$, i.e. $\mathbf{Y}=\left(Y_{(1, n, \widetilde{m}, k)}, \ldots, Y_{(n, n, \tilde{m}, k)}\right)$. First, we will prove that $\mathbf{Y}$ follows a conditional multivariate exponential distribution. From Theorem 3.10 in Kamps [20], the normalized spacings of $\mathbf{Y}$ are i.i.d. exponential distributions with $\lambda=1$, i.e. $\mathbf{D}=$ $(D(1, n, \widetilde{m}, k), \ldots, D(n, n, \widetilde{m}, k))={ }_{s t} \mathbf{E} \sim M C E\left(\mathbf{I}_{n \times n}, \mathbf{0}_{n}\right)$, where

$$
\begin{aligned}
D(1, n, \widetilde{m}, k) & =\gamma_{1} Y_{(1, n, \widetilde{m}, k)}, \\
D(r, n, \tilde{m}, k) & =\gamma_{r}\left(Y_{(r, n, \widetilde{m}, k)}-Y_{(r-1, n, \widetilde{m}, k)}\right),
\end{aligned}
$$

for $2 \leq r \leq n$. A straightforward computation shows that $\mathbf{D}^{t}=\mathbf{A} \mathbf{Y}^{t}$, where $\mathbf{A}$ is given by

$$
\mathbf{A}=\left(\begin{array}{ccccc}
\gamma_{1} & 0 & 0 & \ldots & 0 \\
-\gamma_{2} & \gamma_{2} & 0 & \ldots & 0 \\
0 & -\gamma_{3} & \gamma_{3} & \ldots & 0 \\
\vdots & \vdots & \vdots & \ddots & \vdots \\
0 & 0 & 0 & \ldots & \gamma_{n}
\end{array}\right)
$$

Then, just considering the inverse matrix, we have that $\mathbf{Y} \sim M C E\left(\mathbf{A}^{-1}, \mathbf{0}_{n}\right)$, where $\mathbf{A}^{-1}$ is given 
by

$$
\mathbf{A}^{-1}=\left(\begin{array}{cccc}
\frac{1}{\gamma_{1}} & 0 & \ldots & 0 \\
\frac{1}{\gamma_{1}} & \frac{1}{\gamma_{2}} & \ldots & 0 \\
\vdots & \vdots & \ddots & \vdots \\
\frac{1}{\gamma_{1}} & \frac{1}{\gamma_{2}} & \ldots & \frac{1}{\gamma_{n}}
\end{array}\right)
$$

Since Theorem 4 in Belzunce et al. [9] and due to the fact that $\mathbf{X}$ and $\mathbf{Y}$ have the same set of parameters, $\mathbf{X} \leq_{m c t} \mathbf{Y}$ holds if, and only if, $X \leq_{c}\left[\geq_{c}\right] E$. By (2), the last condition holds if, and only if, $X$ is IFR [DFR].

As mentioned in the previous section several models of increasingly ordered sequences of failure times can be considered as particular cases of the general model of generalized order statistics. Next we provide some of these models.

Taking $m_{i}=0$ for all $i=1, \ldots, n-1$ and $k=1$, we get the random vector of order statistics from a set of $n$ independent and identically distributed (i.i.d) observations. Taking $m_{i}=-1$ for all $i=1, \ldots, n-1$ and $k=1$, we get that the generalized order statistics correspond to failure times of a unit which is being continuously minimally repaired (see [6]). A life-testing experiment of interest in reliability studies involves $N$ independent and identically distributed random variables placed simultaneously on test and at the time of the $m$ th failure $R_{i}$ surviving units are randomly censored from the test (see [7]). The progressively Type-II censored order statistics arising from such reliability experiment can be obtained from the model of by setting $n=m, m_{i}=R_{i}$ and $k=R_{m}+1$. To finish, we recall the model of order statistics under multivariate imperfect repair (see [32]). Let us consider that $n$ items start to work at the same time 0 , with common distribution $F$. Upon failure, an item undergoes a repair. If $i$ items $(i=0,1, \ldots, n-1)$ have already been scrapped then, with probability $p_{i+1}$, the repair is unsuccessful and the item is scrapped, and with probability $1-p_{i+1}$, the repair is successful and minimal. If we consider the ordered random lifetimes resulting under a minimal repair policy as above, then they are a particular case of the density of generalized order statistics based on $F$ with parameters $k=p_{n}$ and $m_{j}=(n-j+1) p_{j}-(n-j) p_{j+1}-1$.

\section{A computational study for the bivariate case}

Let $\mathbf{X}$ be a bivariate random vector, if we want to prove that $\mathbf{X}$ is mct-IFR, we need to find a random vector $\mathbf{Y} \sim M C E\left(\mathbf{A}_{\mathbf{2} \times \mathbf{2}}, \mathbf{b}_{\mathbf{2}}\right)$, such that $\mathbf{X} \leq_{m c t} \mathbf{Y}$. As we pointed out in Section 2, this is equivalent to study the convexity of the function defined in (6), which using the expression (8) is given by

$$
\begin{aligned}
\boldsymbol{\Phi}_{1}\left(x_{1}\right) & =-a_{11} \log \left(1-F_{X_{1}}\left(x_{1}\right)\right)+b_{1}, \\
\boldsymbol{\Phi}_{2}\left(x_{1}, x_{2}\right) & =-a_{21} \log \left(1-F_{X_{1}}\left(x_{1}\right)\right)-a_{22} \log \left(1-F_{\left(X_{2} \mid X_{1}=x_{1}\right)}\left(x_{2}\right)\right)+b_{2} .
\end{aligned}
$$

Note that the convexity of $\boldsymbol{\Phi}_{1}$ and $\boldsymbol{\Phi}_{\mathbf{2}}$ does not depend on the parameter $a_{11}>0$ neither $b_{1}$ and $b_{2}$. Thereby, we can choose $a_{11}=1, b_{1}=0$ and $b_{2}=0$ without loss of generality. On the other hand, $a_{21}$ and $a_{22}$ play an important role in order to study the convexity of $\boldsymbol{\Phi}_{2}$. A first approach to study whether $\boldsymbol{\Phi}_{2}$ is a convex function is to analyze its Hessian matrix, however this is not always a straightforward task. In this case, we suggest using the expressions (12) and (13) to plot the $\boldsymbol{\Phi}_{1}$ and $\boldsymbol{\Phi}_{2}$ functions. Of course, $F_{X_{1}}$ and $F_{\left(X_{2} \mid X_{1}=x_{1}\right)}$ are not always available, therefore we propose to estimate them numerically from the joint probability density function. 
Let us analyze the mct-IFR property for some bivariate random vector. From these examples, we consider the numerical method a feasible path to study the mct-IFR condition for bivariate case, thereby we provide the R-code which let us calculate the $F_{X_{1}}$ and $F_{\left(X_{2} \mid X_{1}=x_{1}\right)}$ functions and $\boldsymbol{\Phi}_{1}$ and $\boldsymbol{\Phi}_{2}$ plots in Arriaza et al. [1].

\subsection{Bivariate normal distributions}

Let $\mathbf{X} \sim N\left(\mu_{\mathbf{2}}, \mathbf{\Sigma}_{\mathbf{2} \times \mathbf{2}}\right)$ be a bivariate Normal distribution and $\mathbf{Y} \sim M C E\left(\mathbf{A}_{\mathbf{2} \times \mathbf{2}}, \mathbf{b}_{\mathbf{2}}\right)$ a bivariate conditional exponential distribution. The parameters of both distributions are given by

$$
\mathbf{X} \sim N\left(\mu_{\mathbf{2}}, \boldsymbol{\Sigma}_{\mathbf{2} \times \mathbf{2}}\right)\left\{\begin{array} { c } 
{ \mu = \mathbf { 0 } \in \mathbb { R } ^ { 2 } } \\
{ \boldsymbol { \Sigma } = ( \begin{array} { l l } 
{ 1 } & { 0 } \\
{ 0 } & { 1 }
\end{array} ) }
\end{array} \quad \text { and } \mathbf { Y } \sim M C E \left(\mathbf { A } _ { \mathbf { 2 } \times \mathbf { 2 } , \mathbf { b } _ { \mathbf { 2 } } ) } \quad \left\{\begin{array}{l}
\mathbf{A}=\left(\begin{array}{ll}
1 & 0 \\
2 & 1
\end{array}\right) \\
\mathbf{b}=\mathbf{0} \in \mathbb{R}^{2}
\end{array}\right.\right.\right.
$$

The joint probability density functions of both random vectors are given by (14) and (15) and their plots are shown in Figure 1.

$$
\begin{aligned}
& f_{\mathbf{X}}\left(x_{1}, x_{2}\right)=\frac{1}{2 \pi} \exp \left\{-\frac{x_{1}^{2}+x_{2}^{2}}{2}\right\}, \quad\left(x_{1}, x_{2}\right) \in \mathbb{R}^{2} \\
& f_{\mathbf{Y}}\left(y_{1}, y_{2}\right)=\frac{1}{a_{11} a_{22}} \exp \left\{\left(\frac{a_{21}}{a_{11} a_{22}}-\frac{1}{a_{11}}\right) y_{1}-\frac{1}{a_{22}} y_{2}\right\} \text {, } \\
& \left(y_{1}, y_{2}\right) \in D=\left\{(x, y) \in \mathbb{R}^{2}: y \geq \frac{a_{21}}{a_{11}} x\right\}
\end{aligned}
$$
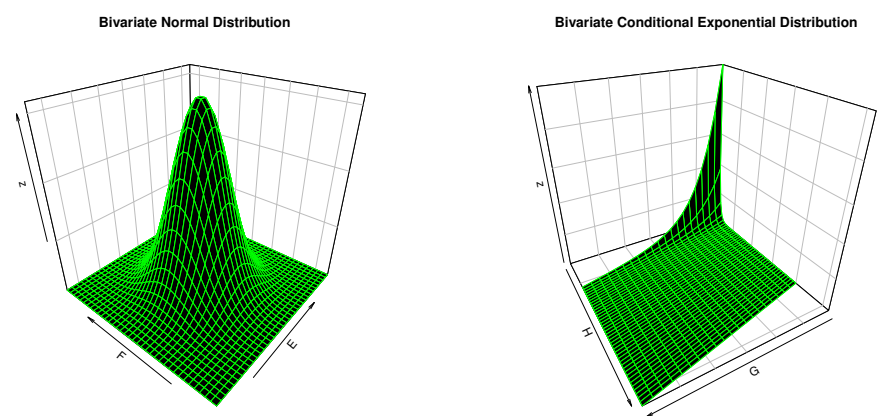

Figure 1: Jpdf of $\mathbf{X}$ and $\mathbf{Y}$ random vectors.

In Figure 2, plots of both component functions of $\boldsymbol{\Phi}$ are shown. The convex shape of these plots graphically reinforces the result obtained in Theorem 2 . 

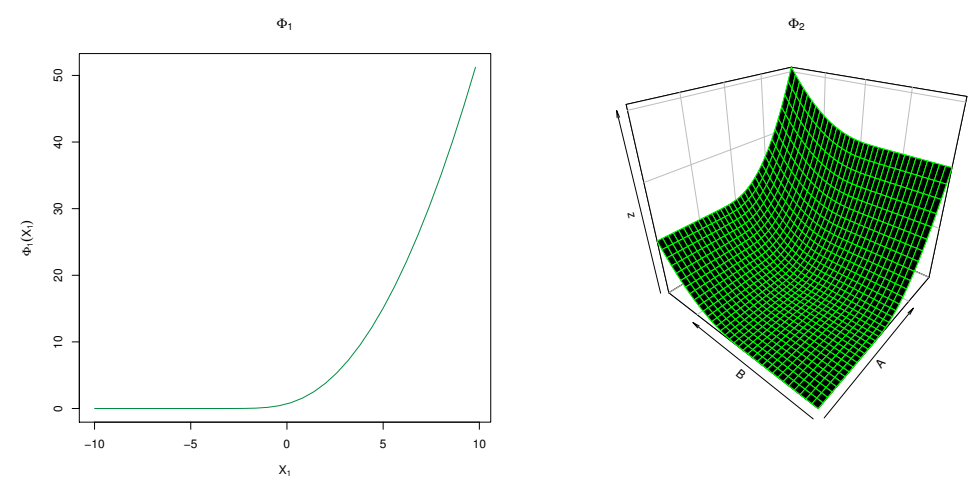

Figure 2: Plots of $\boldsymbol{\Phi}_{1}$ and $\boldsymbol{\Phi}_{2}$ function.

\subsection{Bivariate conditional beta distributions}

This example deals with a random vector $\mathbf{X}$ for what the mct-IFR condition is hard to verify analytically. Let us consider a particular case of a distribution family of which conditional distributions belong to exponential family, see Chapter 4 in Arnold et al. [4]. The general model is given by

$$
f(x, y)=r_{1}(x) r_{2}(y) \exp \left\{\mathbf{q}_{\mathbf{1}}(\mathbf{x})^{t} \mathbf{M} \mathbf{q}_{\mathbf{2}}(\mathbf{y})\right\}
$$

where $\mathbf{q}_{\mathbf{1}}(x)=\left(1 q_{11}(x) \ldots q_{1 l_{1}}(x)\right)^{t}$ and $\mathbf{q}_{\mathbf{2}}(y)=\left(1 q_{21}(y) \ldots q_{2 l_{2}}(y)\right)^{t}$, with the $\mathbf{q}_{\mathbf{k}}$ components linearly independent for all $k \in\{1,2\} ; \mathbf{M} \in \mathbb{M}_{l_{1} \times l_{2}}(\mathbb{R}) ; r_{1}, r_{2}, q_{1 i}, q_{2 j}$ are real functions for all $i \in\left\{1, \ldots, l_{1}\right\}$ and all $j \in\left\{1, \ldots, l_{2}\right\}$.

If we fix the following parameters

$$
\begin{gathered}
r_{1}(x)=\frac{1}{x(1-x)}, \quad r_{2}(y)=\frac{1}{y(1-y)} \\
\mathbf{q}_{\mathbf{1}}(x)=(1 \log (x) \log (1-x))^{t}, \mathbf{q}_{\mathbf{2}}(\mathbf{y})=(1 \log (y) \log (1-y))^{t}
\end{gathered}
$$

then, we obtain the bivariate beta conditional family, denoted by $B C_{2}\left(\mathbf{M}_{\mathbf{3} \times \mathbf{3}}\right)$. On the other hand, let $\mathbf{Y} \sim M C E\left(\mathbf{A}_{\mathbf{2} \times \mathbf{2}}, \mathbf{b}_{\mathbf{2}}\right)$ be a bivariate conditional exponential distribution. The parameters for both distributions are given by

$$
\mathbf{X} \sim B C_{2}\left(\mathbf{M}_{\mathbf{3} \times \mathbf{3}}\right), \quad \mathbf{M}=\left(\begin{array}{ccc}
3.84 & 1 & 1 \\
1 & -1 & -1 \\
1 & -3 & -1
\end{array}\right) \text { and } \mathbf{Y} \sim M C E\left(\mathbf{A}_{\mathbf{2} \times \mathbf{2}}, \mathbf{b}_{\mathbf{2}}\right)\left\{\begin{array}{l}
\mathbf{A}=\left(\begin{array}{ll}
1 & 0 \\
3 & 2
\end{array}\right) \\
\mathbf{b}=\mathbf{0} \in \mathbb{R}^{2}
\end{array}\right.
$$

The joint probability density functions of both random vectors are given in (16) and (17) and 
their plots are given in Figure 3.

$$
\begin{aligned}
f_{\mathbf{X}}\left(x_{1}, x_{2}\right)= & \frac{1}{x_{1}\left(1-x_{1}\right) x_{2}\left(1-x_{2}\right)} \exp \left\{m_{22} \log \left(x_{1}\right) \log \left(x_{2}\right)\right. \\
& +m_{23} \log \left(x_{1}\right) \log \left(1-x_{2}\right)+m_{32} \log \left(1-x_{1}\right) \log \left(x_{2}\right) \\
& +m_{33} \log \left(1-x_{1}\right) \log \left(1-x_{2}\right)+m_{21} \log \left(x_{1}\right)+m_{31} \log \left(1-x_{1}\right) \\
& \left.+m_{12} \log \left(x_{2}\right)+m_{13} \log \left(1-x_{2}\right)+m_{11}\right\}, \quad 0<x_{1}, x_{2}<1 \\
f_{\mathbf{Y}}\left(y_{1}, y_{2}\right)= & \frac{1}{a_{11} a_{22}} \exp \left\{\left(\frac{a_{21}}{a_{11} a_{22}}-\frac{1}{a_{11}}\right) y_{1}-\frac{1}{a_{22}} y_{2}\right\}, \\
& \left(y_{1}, y_{2}\right) \in D=\left\{(x, y) \in \mathbb{R}^{2}: y \geq \frac{a_{21}}{a_{11}} x\right\} .
\end{aligned}
$$
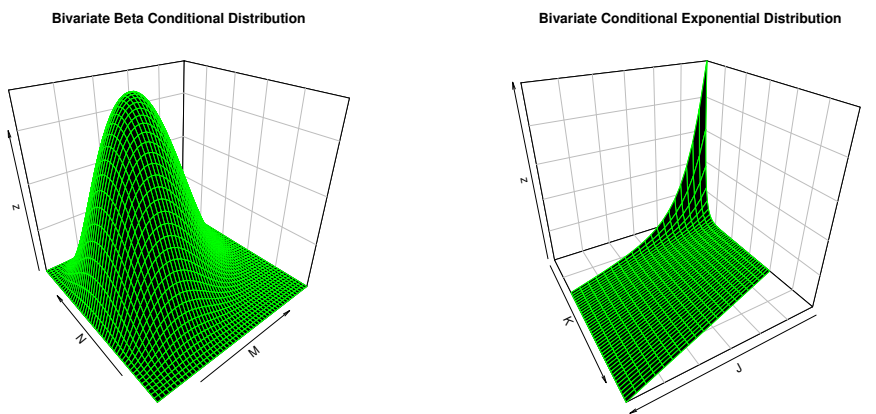

Figure 3: Jpdf of $\mathbf{X}$ and $\mathbf{Y}$ random vectors.
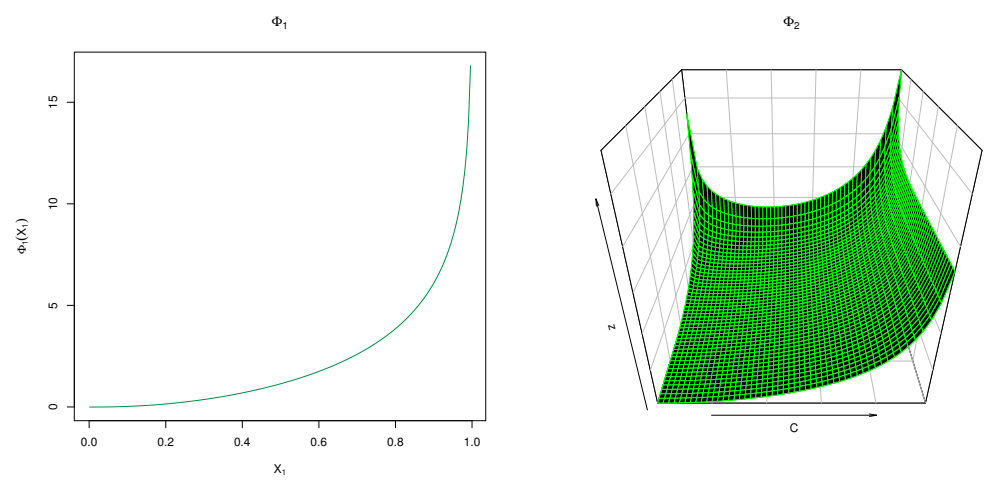

Figure 4: Plots of $\boldsymbol{\Phi}_{1}$ and $\boldsymbol{\Phi}_{2}$ function.

From Figure 4, we observe again that $\boldsymbol{\Phi}_{1}$ and $\boldsymbol{\Phi}_{2}$ have a convex shape, this fact graphically 
suggests that the random vector $\mathbf{X}$ could be mct-IFR. Of course, it must be confirmed by any analytic method.

\section{Conclusions}

Ageing notions play an important role in reliability, survival analysis and other research fields, because they describe how a component or system improves or deteriorates with age. For example, from ageing notions, bounds for survival functions or moment inequalities may be derived. In literature, an abundance of lifetime distributions describing ageing have been considered. Among others, we focus on the IFR (and DFR) notion, that is, distributions having increasing (or decreasing) hazard rate function.

The univariate IFR definition has a clear interpretation and provides the basis for many useful results, which apply when dealing with the analysis of a single unit or of several units with stochastically independent lifetimes. The key of this work is that the IFR ageing of a random variable can be characterized by comparisons with an exponential distribution in the univariate convex transform order proposed by van Zwet [35].

Taking into account the previous characterization of the univariate IFR notion in terms of the univariate convex transform order, we have provided a new multivariate IFR [DFR] notion, the so-called mct-IFR [mct-DFR], comparing a random vector with a properly defined multivariate exponential distribution in a recent generalization of the convex transform order to the multivariate case proposed by Belzunce et al. [9]. This order and, consequently, this new IFR notion are based in the convexity of a well-known transformation which maps a random vector onto another.

In short, a multivariate exponential distribution and a multivariate generalization of the IFR ageing notion is introduced. Properties and relationships of this notion are given, as well as examples dealing with some known probabilistic models related to engineering. A computational study for the bivariate case, and the used R-code that allow us to obtain the plots shown along the paper, is also provided.

\section{Acknowledgements}

The authors want to acknowledge the comments by two anonymous referees, and the Editor of the journal which have improved significantly the presentation of this paper. Félix Belzunce and Julio Mulero acknowledge support received from the Ministerio de Economía y Competitividad (Spain) under grant MTM2012-34023-FEDER. Alfonso Suárez-Llorens and Antonio Arriaza acknowledge support received from the Ministerio de Economía y Competitividad (Spain) under grant MTM2014-57559-P.

\section{References}

[1] Arriaza, A., Belzunce, F., Mulero, J., Suárez-Llorens, A., 2015. An R script to study a new increasing failure rate ageing notion for the bivariate case. Online available at http://hdl.handle.net/10498/17512.

[2] Arias-Nicolás, J., Belzunce, F., Núñez-Barrera, O., Suárez-Llorens, A., 2009. A multivariate IFR notion based on the multivariate dispersive ordering. Applied Stochastic Models in Business and Industry 25, 339-358. 
[3] Arjas, E., Lehtonen, T., 1978. Approximating many server queues by means of single server queues. Mathematics of Operations Research 3, 205-223.

[4] Arnold, B., Castillo, E., Sarabia, J., 1999. Conditional Specification of Statistical Models. Springer Series in Statistics, New York.

[5] Arnold, B., Groeneveld, R., 1995. Measuring skewness with respect to the mode. The American Statistician $49,34-38$.

[6] Ascher, H., and Feingold, H. (1984). Repairable Systems Reliability. Marcel Decker, New York, NY.

[7] Balakrishnan, N. (2007). Progressive censoring methodology: An appraisal (with discussions). Test, 16, 211-296.

[8] Barlow, R., Proschan, F., 1975. Statistical Theory of Reliability and Life Testing, second edition Edition. Holt, Rinehart and Winston, Inc., New York.

[9] Belzunce, F., Mulero, J., Ruíz, J., Suárez-Llorens, A., 2015. On relative skewness for multivariate distributions. Test, DOI:10.1007/s11749-015-0436-4.

[10] Belzunce F, Ruiz J.M., Suárez-Llorens A., 2008. On multivariate dispersion orderings based on the standard construcion. Statistics and Probability Letters 78, 271-281.

[11] Belzunce, F., Shaked, M., 2008a. Stochastic orders and aging notions. In: Ruggeri, F., Faltin, F., Kenett, R. (Eds.), Encyclopedia of Statistics in Quality and Reliability. Wiley, London, pp. $1931-1935$.

[12] Belzunce, F., Shaked, M., 2008b. Multivariate stochastic orders and aging. In: Ruggeri, F., Faltin, F., Kenett, R. (Eds.), Encyclopedia of Statistics in Quality and Reliability. Wiley, London, pp. 1224-1227.

[13] Block, H., Savits, T., 1981. Multivariate classes of life distributions in reliability theory. Mathematics of Operations Research 6, 453-461.

[14] Bryson, M., Siddiqui, M., 1969. Some criteria for ageing. Journal of the American Statistical Association 64, 1472-1483.

[15] Fernández-Ponce, J.M., Pellerey, F., Rodríguez-Griñolo, R. 2011a. A characterization of the multivariate excess wealth ordering. Insurance: Mathematics and Economics 49, 410-417.

[16] Fernández-Ponce, J.M., Pellerey, F., Rodríguez-Griñolo, R. 2011b. On a new NBUE property in multivariate sense: An application. Computational Statistics and Data Analysis 55, 32833294 .

[17] Fernández-Ponce, J., Suárez-Llorens, A., 2003. A multivariate dispersion ordering based on quantiles more widely separated. Journal of Multivariate Analisys 85, 40-53.

[18] Freund, R.J., 1961. A bivariate extension of the exponential distribution. Journal of the American Statistical Association 56, 971-977. 
[19] Johnson, N., Kotz, S., 1975. A vector multivariate hazard rate. Journal of Multivariate Analysis $5,53-66$.

[20] Kamps, U., 1995a. A concept of generalized order statistics. Journal of Statistical Planning and Inference 48, 1-23.

[21] Kamps, U., 1995b. A Concept of Generalized Order Statistics. B.G. Taubner, Stuttgart.

[22] Lai, C., Xie, M., 2006. Stochastic Ageing and Dependence for Reliability. Springer, New York.

[23] MacGillivray, H., 1986. Skewness and asymmetry: measures and orderings. The Annals of Statistics 14, 994-1011.

[24] Mulero, J., 2012. Multivariate comparisons of random vectors with applications. PhD thesis. Universidad de Murcia. From: http://www.tdx.cat/handle/10803/96094.

[25] O'Brien, G., 1975. The comparison method for stochastic processes. The Annals of Probability $3,80-88$.

[26] Rinne, M., 2009. The Weibull Distribution: A Handbook. CRC Press, Boca Raton.

[27] Rosenblatt, M., 1952. Remarks on a multivariate transformation. Annals of Mathematical Statistics 23, 470-472.

[28] Roy, D., 1994. Classification of life distributions in multivariate models. IEEE Transactions on Reliability 43, 224-229.

[29] Roy, D., 2002. Classification of multivariate life distributions based on partial ordering. Probability in Engineering and Information Sciences 16, 129-137.

[30] Rüschendorf, L., 1981. Stochastically ordered distributions and monotonicity of the OCfunction of sequential probability ratio tests. Mathematische Operationsforschung und Statistik Series Statistics 12, 327-338.

[31] Savits, T., 1985. A multivariate IFR class. Journal of Applied Probability 22, 197-204.

[32] Shaked, M. and Shanthikumar (1986), Multivariate imperfect repair. Operations Research, 34, 437-448.

[33] Shaked, M., Shanthikumar, J., 2015. Multivariate conditional hazard rate functions - an overview. Applied Stochastic Models in Business and Industry 31, 285-296.

[34] Spizzichino, F., 2001. Subjective Probability Models for Lifetimes. Chapman and Hall/CRC, New York.

[35] Van Zwet, W., 1964. Convex Transformations of Random Variables. Mathematical Centre Tracts, Amsterdam. 